\title{
Performance Studies of Narrow-Beam Trisector Cellular Systems
}

\author{
Li-Chun Wang, ${ }^{1}$ Kapil Chawla, ${ }^{1}$ and Larry J. Greenstein ${ }^{1}$
}

\begin{abstract}
Early cellular architectures consisted of base stations with omnidirectional antennas. Although directional antennas are widely used in current cellular/PCS (Personal Communication System) the system architecture is often still based upon an omnicell structure, and potential advantages of sectorization are not fully exploited. This paper analyzes and compares two different approaches to sectorization: the traditional wide-beam trisector cell (WBTC) and the newer (but not well characterized) narrow-beam trisector cell (NBTC). Unlike the WBTC architecture using three $100^{\circ}-120^{\circ}$ antennas in a base station, the NBTC architecture (also called "clover-leaf") uses three $60^{\circ}-70^{\circ}$ antennas at each base. We have obtained new results on the quantitative performance of both architectures, taking into account the impact of (1) actual (rather than ideal) antenna patterns, (2) channel utilization factor, (3) site diversity, (4) nonideal cell site locations, and (5) the influences of reuse factor and shadow fading. We show that NBTC system performance exceeds that of WBTC systems, both in signal-to-interfer ence ratio (SIR) statistics and signal coverage. The improvement in coverage translates to a reduced power requirement of about $2 \mathrm{~dB}$ for the same cell size, or equivalently, to a $25 \%$ larger cell coverage area for the same power. The improvement in SIR performance corresponds to a gain of $2-3 \mathrm{~dB}$ in the 90th SIR percentile. This gain can be translated to significantly improved grade-of-service in cellular environments, with no attendant cost.
\end{abstract}

\section{INTRODUCTION}

Sectorization techniques are widely used in cellular systems to reduce co-channel interference $(\mathrm{CCI})$ by means of directional antennas. Because of the reduced interference, sectored cellular systems can allow a more efficient frequency reuse plan. This paper analyzes and compares two different approaches to sectorization: a traditional, well-studied approach and a newer, less-studied approach.

Cellular system planning usually requires defining a hypothetical cell shape. In conventional cellular systems with omnidirectional antennas, contours of equal received power tend to be approximately circular. Since a hexagon is a close approximation to a circle, and hexagons tessellate, the cell shape is assumed to be

\footnotetext{
${ }^{1}$ AT\&T Laboratories-Research, Red Bank, New Jersey 07701.
}

hexagonal. Therefore, site layout diagrams are usually formed as a hexagonal grid. For sectored cellular systems with directional antennas, two distinct methods are adopted to define cell contours. In one, as in the omnidirectional case, a hexagon is used to describe the coverage contour for a base station composed of multiple directional antennas. The coverage area of each antenna is determined by dividing the hexagon among the number of antennas. In other words, this approach restricts the sector coverage area of a directional antenna to be a subarea of a hexagon. Based on this method, traditional trisector cellular systems (as shown in Fig. 1B) use three $100^{\circ}-120^{\circ}$ antennas at a base station. We call this kind of cell the wide-beam trisector cell (WBTC). In contrast, the second method incorporates the radiation pattern of a directional antenna to produce a more realistic sector coverage, rather than dividing a hexagon 


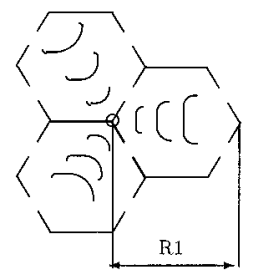

(a)

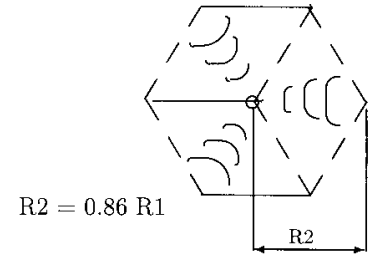

(b)
Narrow-Beam Trisector Cell

Wide-Beam Trisector Cell

Fig. 1. Hypothetical cell contours of the NBTC and WBTC systems.

as in the first approach. The cellular contour is then defined by connecting the coverage areas of each directional antenna. Clover-leaf-shaped cells, as shown in Fig. $1 \mathrm{~A}$ and reported in [1], are obtained by this method. In this paper, we call the clover-leaf cell a narrow-beam trisector cell (NBTC) because three $60^{\circ}-70^{\circ}$ directional antennas are used at a base station.

Intuitively, we can see that the WBTC approach may result in poor coverage and severe interference due to the difficulty of matching the subarea of a hexagon to the coverage area of a directional antenna. Ideally, each sector in a WBTC system is assumed to cover a diamond-shaped area (Fig. 1B), but the actual coverage area formed by the directional antenna differs significantly from this shape. On the other hand, an NBTC system can be expected to have better performance, because the ideal sector coverage area more closely matches the actual coverage area formed by the directional antenna (Fig. 1A). However, detailed performance studies of NBTC systems are lacking in the literature. Even for WBTC systems, moreover, most papers assume ideal antenna patterns and ideal cell site locations [2].

This paper presents new results on the quantitative performance of NBTC and WBTC systems, taking into account the impact of actual radio patterns of directional antennas and location variations of the cell sites. Furthermore, we address several important issues associated with the effects of shadowing, cell loading, and site diversity. We show that the NBTC system can improve both interference and signal coverage performance while using the same cell sites, that is, without extra infrastructure cost.

The remainder of the paper is organized as follows: Section 2 describes the propagation model. The cell contour and site layout of NBTC and WBTC systems are introduced in Section 3. Section 4 illustrates the signalto-interference (SIR) performance, and Section 5 illustrates the signal coverage performance, both of them for the baseline case of ideal cell site locations and no site diversity. The impact of site diversity and of site location variation are quantified in Sections 6 and 7, respectively.

\section{PROPAGATION MODEL}

The cellular radio propagation environment is complex and highly random. It is generally characterized by three effects: median path loss, shadowing, and multipath amplitude fading. For system-level studies like the present one, the channel power response is usually averaged over multipath fading.

\subsection{Median Path Loss}

Let $P_{r}$ be the local spatial average of received power at a terminal located at distance $d$. The ratio of $P_{r}$ to $P_{t}$ (the transmit power) is called the local mean attenuation or path loss. The variation of path loss (or of $P_{r}$ ) with $d$ tends to be monotonically decreasing; in reality, however, it has a statistical range of values at any $d$ because of shadowing. What we can say is that the median of this statistical spread can be modeled by the monotonic relationship

$$
P_{\text {med }}=\frac{C P_{t} G(\theta)}{d^{\gamma}}
$$

where $G(\theta)$ is the composite transmit-receive antenna gain at angle $\theta^{1} ; \gamma$ is the path loss exponent; and $C$ is a constant that includes the effects of antenna heights, terrain type, etc. The statistical variation about this median due to shadowing tends to be lognormal, as we discuss next.

\subsection{Shadow Fading}

To characterize the shadowing effect, a lognormal distribution is used to describe the statistical variation of local mean power about the median. That is, if $\Omega$ is the $\mathrm{dB}$ value of local mean received power, then the probability density function (pdf) of $\Omega$ is

$$
f_{\Omega}(\Omega)=\frac{1}{\sqrt{2 \pi} \sigma} \exp \left[\frac{-\left(\Omega-10 \log P_{\text {med }}\right)^{2}}{2 \sigma^{2}}\right]
$$

where the shadowing spread $\sigma$ is typically in the range 6-12 dB [3] and $P_{m e d}$ is given by (1). 


\section{NOMINAL CELL COVERAGE AND SITE LAYOUT}

\subsection{Analytical Model}

We define the cell boundary as the contour with the same median received power from the base station. From (1), the propagation distance, $d_{S}$, at which the median received power from a single sector is equal to $S$, can be obtained as follows:

$$
d_{S}=G(\theta)^{1 / \gamma} \times\left(\frac{C P_{t}}{S}\right)^{1 / \gamma}
$$

where azimuth angle $\theta$ is with respect to the direction of maximum antenna gain, $\theta=0$. From (3), it can be observed that the contour shape of a sector, and therefore also the cell, is determined by the radiation pattern of the directional antenna and the exponent $\gamma$, while the other parameters influence the cell size. By applying an actual antenna radiation pattern in (3), we can verify the accuracy of the hypothetical cellular contours of WBTC and NBTC shown in Fig. 1.

\subsection{Cell Contours}

Consider a WBTC system employing three directional antennas with a $120^{\circ}$ half-power beam width (HPBW) at the base station. From (6-91) of [4], we plot the radiation pattern of an array antenna with $120^{\circ}$ HPBW in Fig. 2. Substituting the antenna gain pattern of Fig. 2 into (3), we obtain Fig. 3, which shows a sector contour of a WBTC for three values of $\gamma$. Comparing the hypothetical sector boundary (solid line) with the actual contours (nonsolid lines) we observe two major drawbacks of WBTC systems. First, side-lobe levels (i.e., gains at angles larger than the HPBW) are significant in adjacent sectors. This may cause severe adjacent channel interference in the same cell and co-channel interference in other cells. Second, the corners of the hexagon at the boundary between two sectors of a cell may not be well covered because of the $3 \mathrm{~dB}$ lower antenna gain as compared to the antenna main-beam direction.

Using the same method as for a WBTC, we plot the radiation pattern of a $60^{\circ}$ (HPBW) antenna [4] and a sector contour for an NBTC in Figs. 4 and 5, respectively. From Fig. 5, we observe that the hexagonal sector shape closely matches the contour of a $60^{\circ}$ antenna for realistic values of path loss exponent. It is interesting to note that at an angle of $60^{\circ}$ from the main-beam direction,
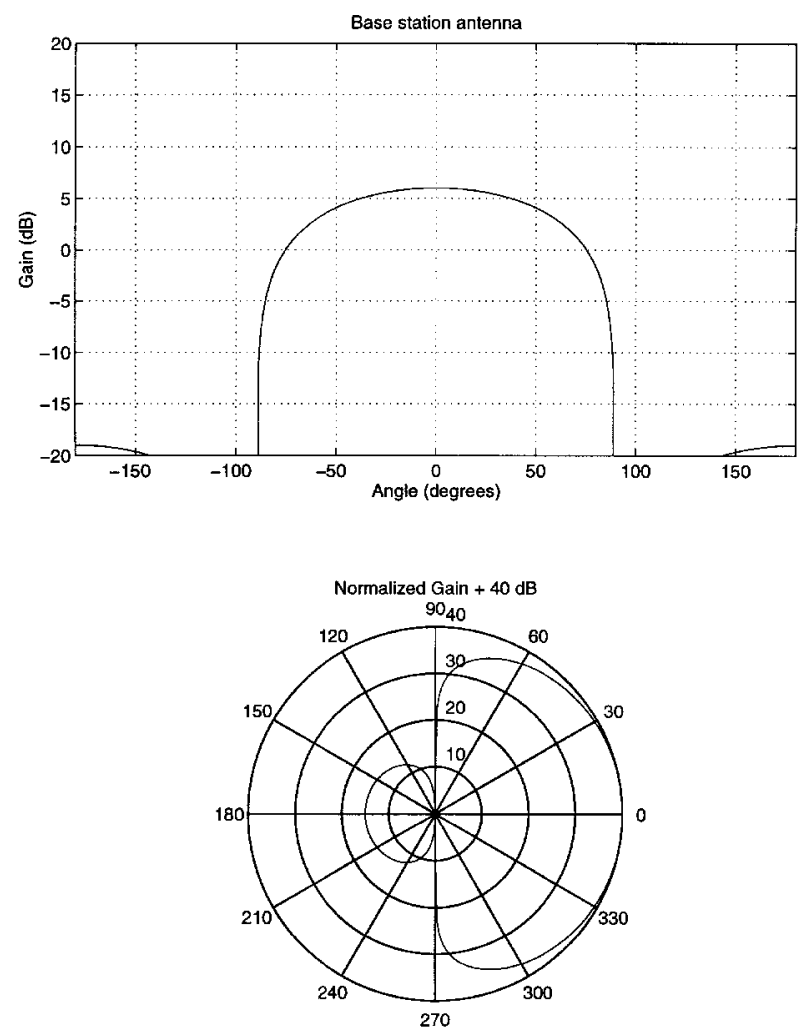

Fig. 2. Horizontal pattern with $120^{\circ}$ beam width (WBTC).

the antenna gain is about $10 \mathrm{~dB}$ lower than the maximum antenna gain; however, the distance is one half of the cell radius. Assuming an inverse fourth-power path

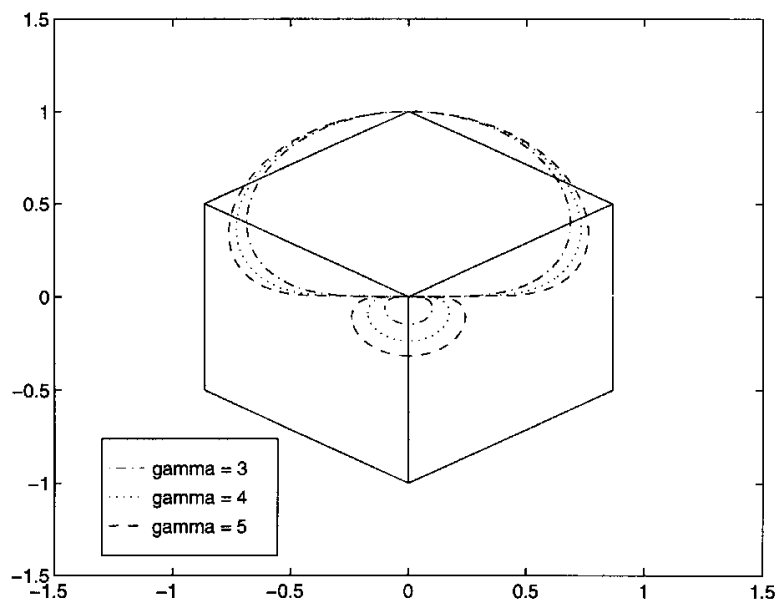

Fig. 3. Sector contour for a wide-beam trisector cell (WBTC), where the solid line represents the hypothetical results and nonsolid lines are calculated results for different values of path loss exponents $\gamma$. 

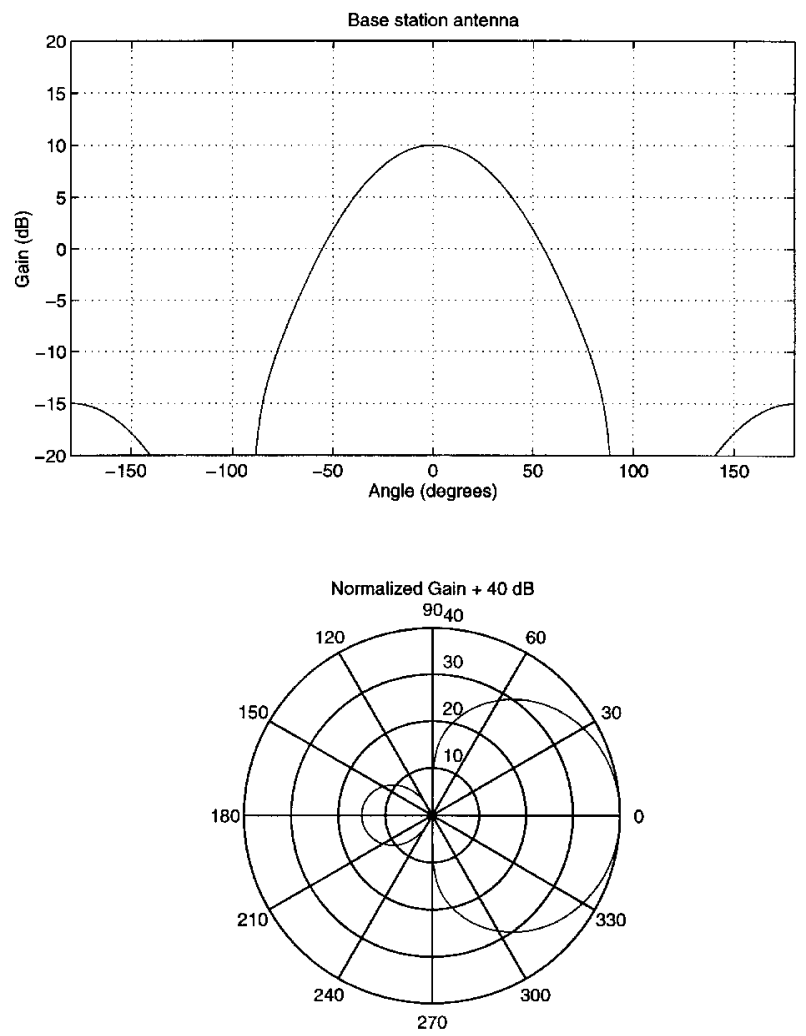

Fig. 4. Horizontal pattern with $60^{\circ}$ beam width (NBTC).

loss model $(\gamma=4)$, we obtain a distance gain at this point of about $12 \mathrm{~dB}$, which can effectively compensate the antenna loss of $10 \mathrm{~dB}$.

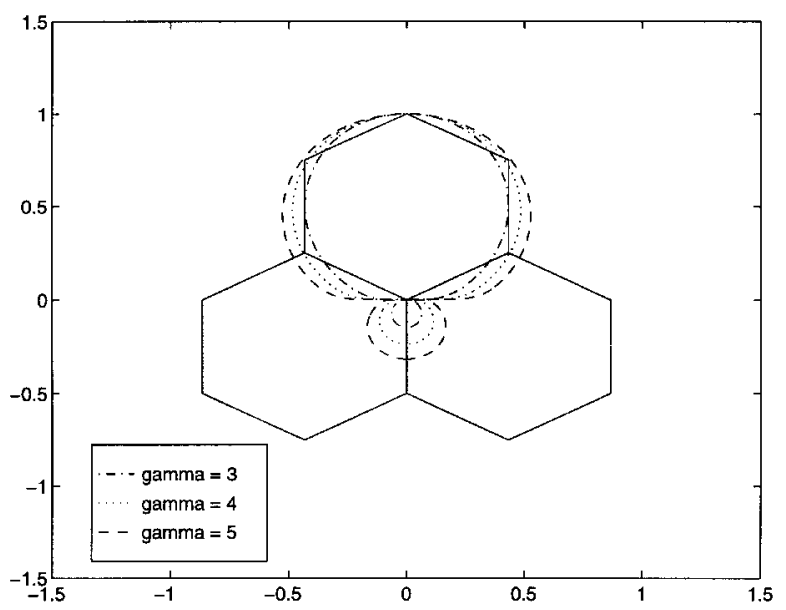

Fig. 5. Sector contour for a narrow-beam trisector cell (NBTC), where the solid line represents the hypothetical results and nonsolid lines are calculated results for different values of path loss exponents $\gamma$.
As a check on modeling accuracy, we have compared the assumed (theoretical) antenna patterns of Figs. 2 and 4 with the measured patterns of real products, e.g., $[5,6,7]$. We find that (1) the assumed patterns match the measured ones fairly well in the front plane (within $\pm 90^{\circ}$ of boresight); (2) the assumed patterns have somewhat lower gains than the measured ones in the back plane; and (3) the latter discrepancy is greater for the wider-beam antennas than for the narrower-beam antennas. Since the impact of interference from back lobes is much less than that from front lobes, we conclude that the patterns of Figs. 2 and 4 will give results that are only slightly optimistic, and a bit more so for WBTC systems than for NBTC systems.

\subsection{Site Layouts}

Site layouts can be obtained by tessellating the service area using the contour of each type of cell. With cell contours as shown in Fig. 1, examples of site layouts for the two types of cells are shown in Figs. 6 and 7. For this example, we assume a reuse factor of $N=3$ for both cases. We also assume that both systems use the same cell site locations. This implies that the cell sites are separated by the same distance $(D)$ for these two systems. From the site layouts in Figs. 6 and 7, we obtain

$$
R_{2}=\frac{\sqrt{3}}{2} R_{1}
$$

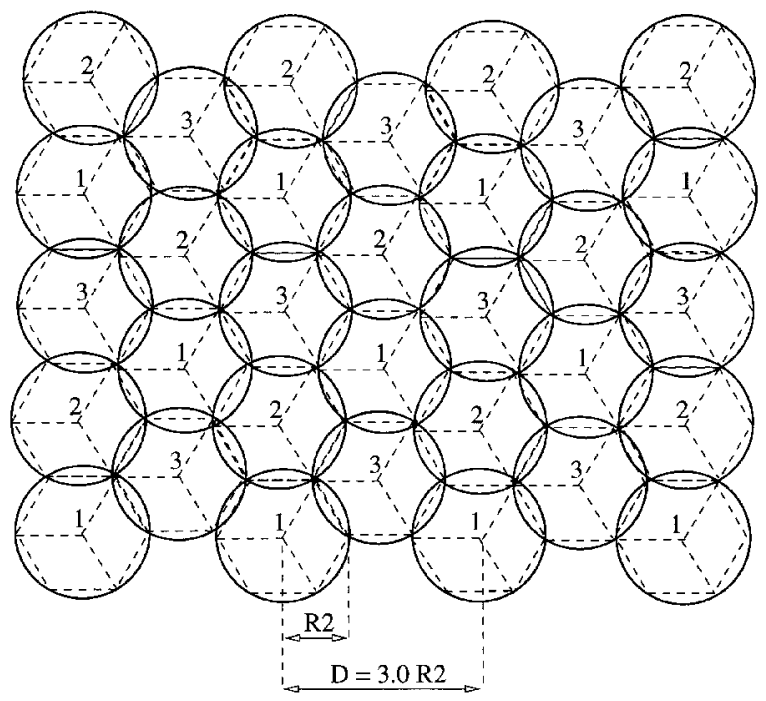

Fig. 6. Site layout for WBTC system with reuse factor $N=3$. 


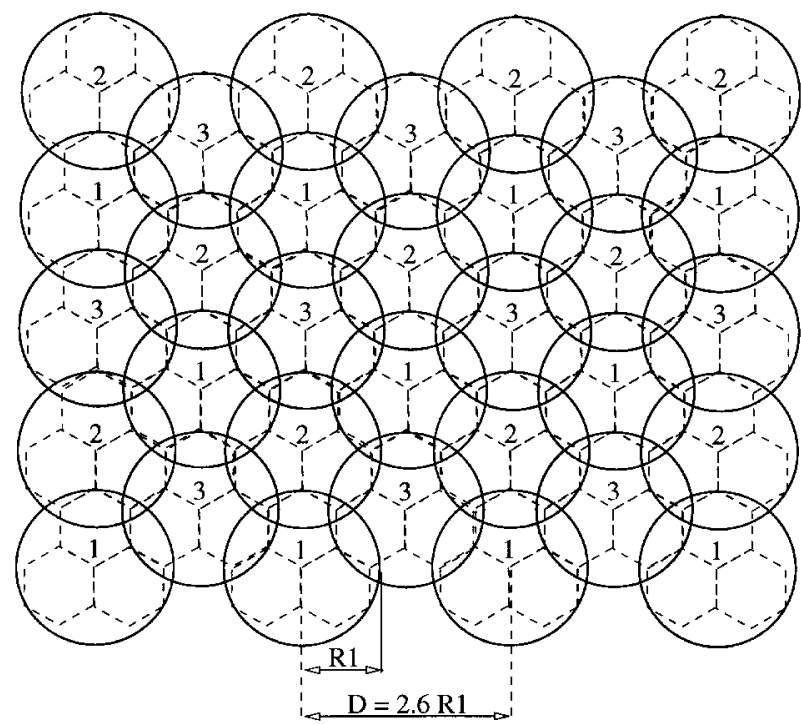

Fig. 7. Site layout for NBTC system with reuse factor $N=3$.

where $R_{1}$ is the radius of an NBTC, and $R_{2}$ is the radius of a WBTC. To maintain the same received signal strength at the farthest points of an NBTC and a WBTC with the same transmitting power, the base station in an NBTC system needs about $2.5 \mathrm{~dB}$ higher antenna gain, according to (4) and assuming $\gamma=4$. This requirement is easily met because most directional antennas with around $60^{\circ} \mathrm{HPBW}$ have $2 \mathrm{~dB}$ higher antenna gain than antennas with around $100^{\circ} \mathrm{HPBW}$. Note that (4) also implies that the area of a sector of an NBTC is equal to that of a WBTC. Clearly, this should be the case because for both layouts the total area is split into an equal number of sectors.

\section{SIR PERFORMANCE}

\subsection{Performance Criteria}

The coverage probability or reliability function is defined to be

$$
P(S I R) \triangleq \operatorname{Prob}[(\text { Signal-to-Interference Ratio })>S I R]
$$

where the signal-to-interference ratio is $S / I ; S$ is the local mean of the desired received power; and $I$ is the local mean of the total received interference power, i.e.,

$$
I=\sum_{i=1}^{n} I_{i}
$$

where $I_{i}$ is the power of the $i$-th individual interferer and $n$ is the number of active co-channel interferers. ${ }^{2}$ This function gives the reliability of service at each possible value of signal-to-interference ratio (SIR), i.e., the fraction of the coverage area over which the SIR exceeds the value. If, for a given air interface, the required (or threshold) value for good reception is $S I R_{\mathrm{th}}$, then $P\left(S I R_{\text {th }}\right)$ is the coverage probability, or reliability, for that interface, and $\left[1-P\left(S I R_{\mathrm{th}}\right)\right]$ is the outage probability. Let $S I R_{90}$ denote the value of $\mathrm{S} / \mathrm{I}$ exceeded $90 \%$ of the time over the coverage area, i.e., $P\left(S I R_{90}\right)=0.9$. Then, a reliability of $90 \%$ or more (outage probability of $10 \%$ or less) is achieved for a given cellular design if $S I R_{90} \geq S I R_{\text {th }}$.

Using (1) and (6), and assuming that all sectors transmit at the same power, the signal-to-interference ratio for a given user can be expressed as

$$
\frac{S}{I}=\left[\sum_{i=1}^{n}\left(\frac{G_{i}\left(\theta_{i}\right)}{G\left(\theta_{0}\right)}\right)\left(\frac{d_{i}}{d_{0}}\right)^{-\gamma}\left(\frac{\lambda_{i}}{\lambda_{0}}\right)\right]^{-1}
$$

where $\lambda$ is the shadow fading variable $(10 \log (\lambda)$ is a Gaussian random variate with zero mean and standard deviation $\sigma$ ), the subscript 0 corresponds to the user (desired signal), and $i=1, \ldots, n$ are the subscripts for the active interferers.

It is worthwhile noting that if an ideal antenna pattern were used (i.e., no side lobes and back lobes), a trisector system with $120^{\circ}$ beam widths would have only two first-tier interferers; however, for any realistic antenna pattern there exists six first-tier interferers, each of which experiences a different antenna gain. We include the impact of side-lobe and back-lobe interference in the study by assuming the more realistic patterns of Figs. 2 and 4.

\subsection{Simulation Model}

To determine coverage probability, the quantities $d_{i}$, $\theta_{i}$, and $\lambda_{i}$ in (7), $i=0, \ldots, n$ are treated as random variables, and the probability distribution of $\mathrm{S} / \mathrm{I}$ is obtained. Since this is prohibitive to do analytically, we use a simu- 
lation platform, invoking the following assumptions and conditions:

1. We consider only the base-to-mobile (downlink) direction. In most cases, this is the performancelimiting direction $[8,9]$ and therefore sufficient for study purposes. This is because the uplink benefits relative to the downlink by virtue of both diversity reception and power control.

2. In conformity with current practice in frequency division multiple access (FDMA) and time division multiple access (TDMA) systems, we do not consider downlink power control.

3. The user locations are randomly generated from a uniform distribution over the service area.

4. The shadow fading components $\left(\lambda_{i}, i=0,1\right.$, $\ldots, n)$ are assumed to be mutually independent. In reality, this may not always be true, since local shadowing for a given user location can affect its paths to all base stations. Some studies have addressed this issue of correlated lognormal fading [10], but the present one does not. On intuitive grounds, we can say that this will make our computed SIR statistics slightly pessimistic.

5. We consider only the first-tier co-channel interferers $(n \leq 6$ in (7)) to simplify the simulations. On average, the total interference from the second tier is $10 \mathrm{~dB}$ or more below that from the first tier, and thus can be either ignored or accounted for via simple adjustments.

6. Initially, we evaluate a fully loaded system, wherein all co-channel interferers in the first tier are simultaneously active $(n=6$ in (7)). Later, we simulate more realistic environments wherein every interferer becomes active randomly, with a fractional utilization over time of $\rho<1$.

7. Initially, our simulations assume that each user communicates with the nearest base, rather than the "best" one. Later, we add site diversity (which is more realistic), wherein each user is paired with the base supplying the strongest signal. By treating both cases, we will be able to quantify and compare the benefits of site diversity for WBTC and NBTC systems.

8. Initially, we assume all cell sites are located on an ideal hexagonal grid. Later, we randomly reposition all cell sites in the service area by distances up to $50 \%$ of a cell radius. This will enable us to quantify and compare the sensitivities of WBTC and NBTC systems to cell site variations.
Our simulation platform, built upon the above assumptions and conditions, was used to conduct thousands of trials. In each trial, the set $\left\{d_{i}, \theta_{i}, \lambda_{i}\right\}, i=0$, $1, \ldots, 6$, was generated randomly and $\mathrm{S} / \mathrm{I}$ was computed using (7). The population of S/I values obtained was then used to compute $\mathrm{P}(S I R),(5)$. This was done for both WBTC and NBTC systems, and for a variety of system and propagation parameters.

\subsection{Numerical Results: Full Loading}

We treat as the "baseline" case a system with full loading (all interferers active), no site diversity (each user terminal is linked to its nearest cell site), and an ideal cellular grid (all cell sites are centered on equisized tessellating hexagons). We will move toward more realistic cases later, as we relax these conditions one by one.

Figure 8 compares $\mathrm{P}(S I R)$ for $\mathrm{NBTC}$ and WBTC systems, and also shows the impact of the reuse factor $(N)$ on performance. ${ }^{3}$ For any given $N$, it is clear that an NBTC system has better SIR performance. As a specific example, an NBTC system with $N=3$ performs better than a WBTC system with $N=4$. Therefore, if the SIR threshold $\left(S I R_{\mathrm{th}}\right)$ is, say $13.0 \mathrm{~dB}$, and the desired reliability is $90 \%$, a fully loaded NBTC system would provide acceptable performance with a $33 \%$ capacity improvement over a corresponding WBTC system.

Based on our simulations, Table I lists the 90th-percentile values of SIR for different values of $N$ and $\sigma$. It is seen that, for a given $N$ and $\sigma$, an NBTC system

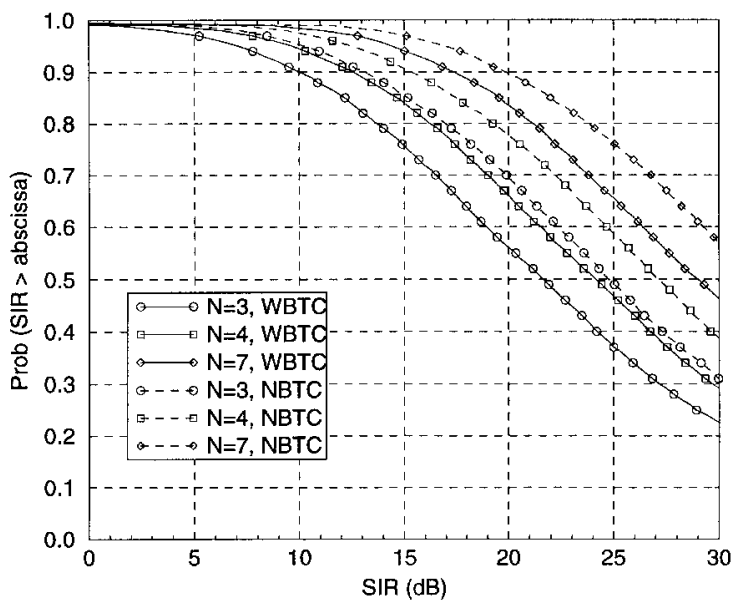

Fig. 8. SIR performance comparison between WBTC systems (solid lines) and NBTC systems (dashed lines) without site diversity, where $N$ is reuse factor, $\sigma=6 \mathrm{~dB}$, and $\rho=1$. 
Table I. The 90th-Percentile SIR for NBTC and WBTC Systems Without Site Diversity

\begin{tabular}{crrrrrrrr}
\hline & \multicolumn{3}{c}{ NBTC } & & \multicolumn{3}{c}{ WBTC } \\
\cline { 2 - 3 } \cline { 6 - 8 }$\sigma(\mathrm{dB})$ & $N=3$ & $N=4$ & $N=7$ & & $N=3$ & $N=4$ & $N=7$ \\
\hline 4 & 17.12 & 19.39 & 23.82 & & 14.55 & 16.18 & 21.69 \\
6 & 13.80 & 16.21 & 20.77 & & 11.11 & 13.55 & 18.29 \\
8 & 10.19 & 12.44 & 17.13 & & 7.10 & 9.38 & 14.28 \\
10 & 6.31 & 8.64 & 13.14 & & 2.62 & 4.98 & 9.78 \\
\hline
\end{tabular}

improves $S I R_{90}$ by 2.1 to $3.7 \mathrm{~dB}$ over a WBTC system, which can translate to a significant gain in system reliability. At the same time, these gains must be viewed with caution. They pertain to the baseline case where each cell site communicates only with terminals in its nominal service area, i.e., each terminal is linked with its nominal serving base rather than the "best" one. When we later add the realism of cell site diversity (Section 6), we will see that the differences in Table I shrink by 1 to $1.5 \mathrm{~dB}$. Moreover, when we add cell site variations (Section 7), they will shrink even further. Figure 9 illustrates the impact of using an "ideal antenna" assumption to evaluate WBTC performance. It is seen that a WBTC design study based on ideal directional antennas may overestimate the SIR performance by as much as 3 $\mathrm{dB}$.

\subsection{Numerical Results: Partial Loading}

The channel utilization of any sector in a cellular system must be less than $100 \%$ to obtain suitably low

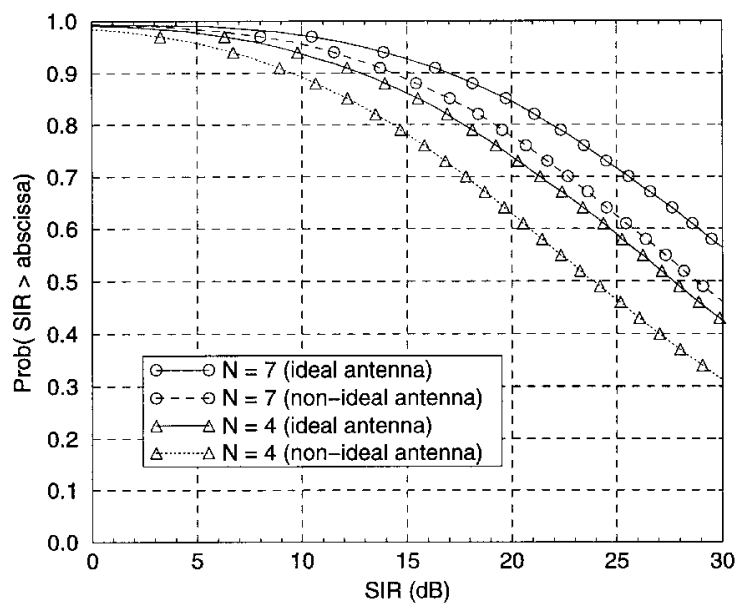

Fig. 9. Effects of nonideal antenna patterns on the SIR performance of WBTC systems, where $\sigma=8 \mathrm{~dB}, \rho=1$, and without site diversity.

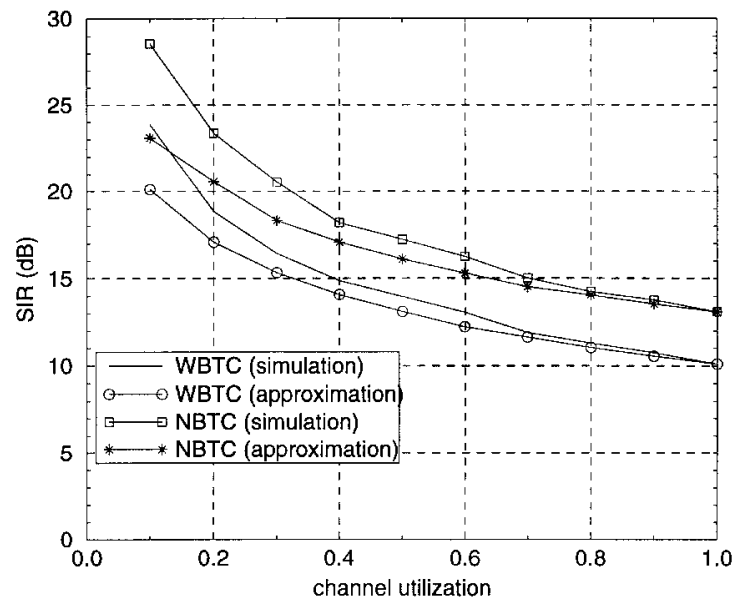

Fig. 10. The simulation and approximation results of the 90th-percentile values of SIR vs. channel utilization for both NBTC and WBTC systems with $N=3$ and $\sigma=6 \mathrm{~dB}$.

call-blocking probabilities. In general, the desired utilization factor $(\rho)$ will depend on the number of channels served in the sector, the traffic statistics, and the callblocking target. For reference, in an AMPS system with $N=7$ and 3 -sectored cells, $\rho \approx 0.65$ for a fully loaded sector with a call-blocking target of $2 \%$. Assuming all sectors in the system have a channel utilization equal to $\rho$, it follows that the average of the total received interference power is also scaled by $\rho$, and thus a first-order approximation to $S I R_{90}$ as a function of $\rho$ is

$$
\operatorname{SIR}_{90}(\rho)=\operatorname{SIR}_{90}(1)-10 \times \log (\rho)
$$

We have tested the above approximate model via our simulations. Figure 10 is a plot of $S I R_{90}$ vs. $\rho$ for both WBTC and NBTC systems with $N=3$ and $\sigma=6 \mathrm{~dB}$. The results predicted by (8) compare favorably with those from simulations over the range $\rho \geq 0$.4. In general, we can say that (8) gives a useful worst case bound on the variation of $S I R_{90}$ (or any other percentile) with $\rho$.

It is worth noting from Fig. 10 that, for $N=3$ and $\sigma=6 \mathrm{~dB}$, an NBTC system yields $\operatorname{SIR}_{90}=17 \mathrm{~dB}$ (as required in current AMPS and IS-136 systems) for $\rho=$ 0.5

\section{COVERAGE PERFORMANCE}

\subsection{Analysis}

Using the simulation platform discussed in Section 4, we have also studied the statistics of the user sig- 
nal level throughout a service area. We did this for both WBTC and NBTC systems, assuming as before that both systems have the same cell site spacings.

In these initial simulations, we have assumed no site diversity and an ideal cellular grid. Our aim is to determine which system needs more transmit power, in this case, to achieve a specified signal coverage.

To begin, the local mean received power can be written as

$$
P_{r}=C+P_{t}-10 \gamma \log (d)+G(\theta)+10 \log (\lambda)
$$

where all terms are as before except that $C$ and $G(\theta)$ are in $\mathrm{dB}$ and $P_{r}$ and $P_{t}$ are in $\mathrm{dBm}$. The last three terms are a function of the user, and so we lump them together as

$$
X=-10 \gamma \log (d)+G(\theta)+10 \log (\lambda)
$$

Recall that $10 \log (\lambda)$ is a zero-mean Gaussian variate with standard deviation $\sigma$. For convenience only, we assume $d$ is in $\mathrm{km}$.

A typical performance requirement is that $P_{r}$ should fall below some minimum value, $P_{0}$, for no more than some percentage, $p$, of the service area (i.e., $p$ is the specified outage probability). Now let $X_{p}$ be the numerical value that $X$ falls below at $p$ percent of locations. From (9) and (10), it is then easy to see that the performance requirement will be met if

$$
P_{t} \geq P_{o}-C-X_{p}
$$

Clearly, the larger $X_{p}$ is, the less transmit power is needed. Thus, by finding the cumulative distribution function (CDF) of $X$ for both systems, we can learn the differences in transmit power requirements for specified values of outage probability.

\subsection{Numerical Results}

Figure 11 shows CDFs of $X$ for both NBTC and WBTC systems for different values of $\sigma$, where the antenna radiation patterns are as shown in Figs. 2 and 4. In general, NBTC systems have larger values of $X$ than WBTC systems at the same probability level. Thus, NBTC systems require less transmit power. At the $p=10 \%$ level, the difference is typically $2.5 \mathrm{~dB}$. The reason is mainly the higher antenna gain and also a better matching of the hypothetical sector shape with the actual sector contour in an NBTC system. We also note from Fig. 11 that required transmit power increases with $\sigma$, as expected.

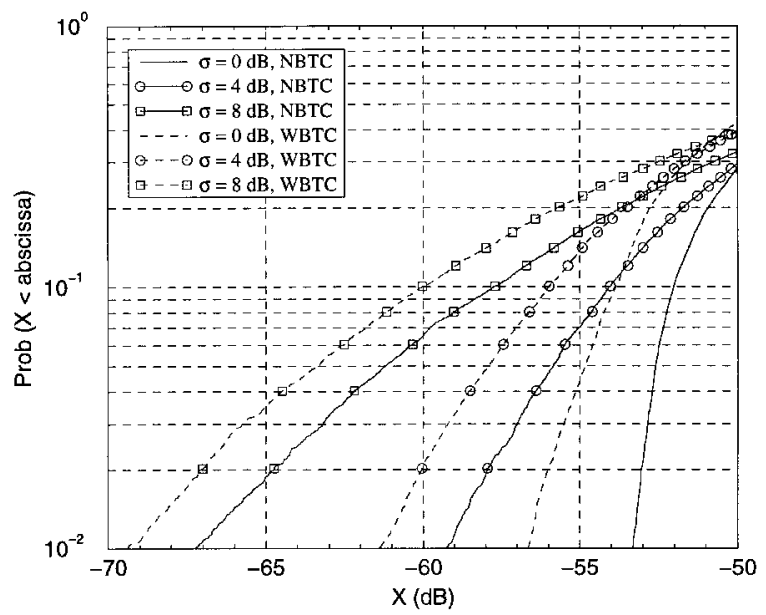

Fig. 11. CDF of $X$, where the cell radius $R_{1}=4 \mathrm{~km}$ and $\gamma=4$.

The impact of path loss exponent $\gamma$ is shown in Fig. 12. Also as expected, we see that as $\gamma$ increases, $X$ decreases, i.e., a higher transmission power is required. More important, we see that the power improvement of NBTC systems over WBTC systems is roughly the same for different values of $\gamma$.

It is also interesting to know whether the better radio signal strength (RSS) performance for NBTC systems implies a stronger interference to others. Figure 13 shows that at a higher level of $\mathrm{CDF}$, say $90 \%$, a user in an NBTC system actually receives less interference power from other co-channel cells. This result is not surprising because the narrower-beam antenna in an NBTC

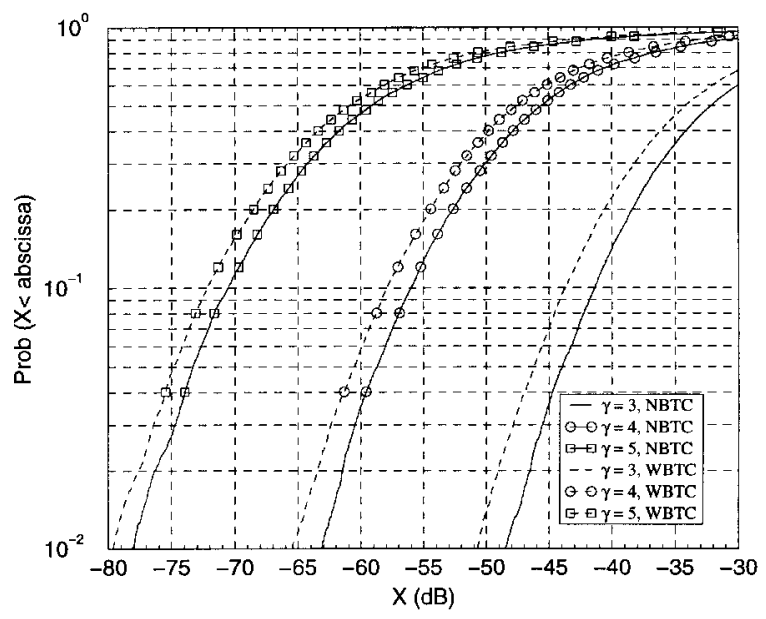

Fig. 12. Effects of path loss exponent $\gamma$ on the $X$, where the cell radius $R_{1}=4 \mathrm{~km}$ and $\sigma=6 \mathrm{~dB}$. 


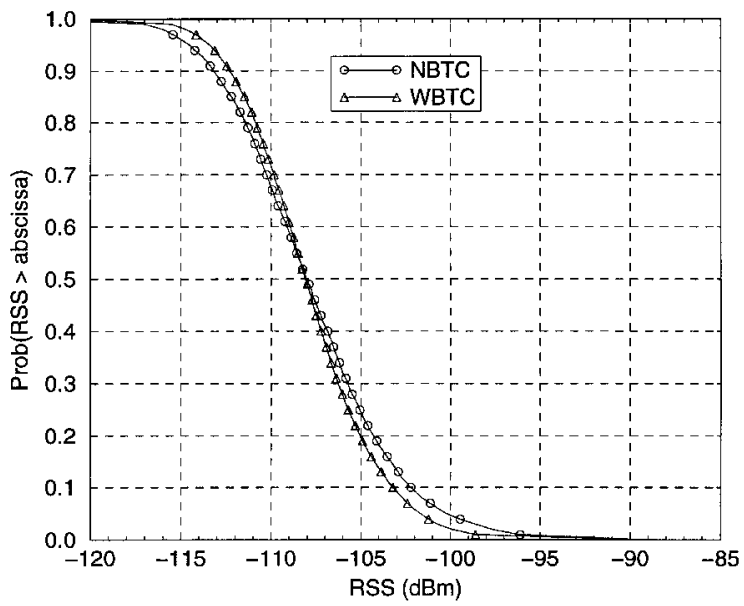

Fig. 13. Comparison of total interference power between NBTC and WBTC systems, where $N=4, P_{t}=45 \mathrm{dBm}$ and $\sigma=6 \mathrm{~dB}$.

system obviously propagates radio energy in a narrower area, resulting in lower interference. These two observations for NBTC systems-less interference and better RSS performance-explain why an NBTC system has better SIR performance.

\section{SITE DIVERSITY}

Unlike the hypothetical cell contours shown in Fig. 1, actual cell coverage areas are determined through the process of site selection. During this process, each mobile terminal compares the received signal power levels from surrounding base stations and then selects the best one to connect to. This technique is called site diversity or macrodiversity. The performance gains that result from site diversity for both the NBTC and WBTC systems are evaluated and compared in this section. For simplicity, we consider the fully loaded case and assume that an ideal desired-power algorithm is used, i.e., only the desired-signal power levels are compared. Although the signal power measured by the mobile station is the summation of the desired signal and any co-channel interference, the performance of the "total-power" algorithm is close to that of the desired-power algorithm for realistic values of SIR [11].

We find, as may be expected, that site diversity improves both coverage performance and SIR performance. We consider SIR performance first. Figure 14 shows this performance with and without site diversity for both NBTC and WBTC systems, for $N=4$ and $\sigma$ $=6 \mathrm{~dB}$. Observe that the NBTC system outperforms

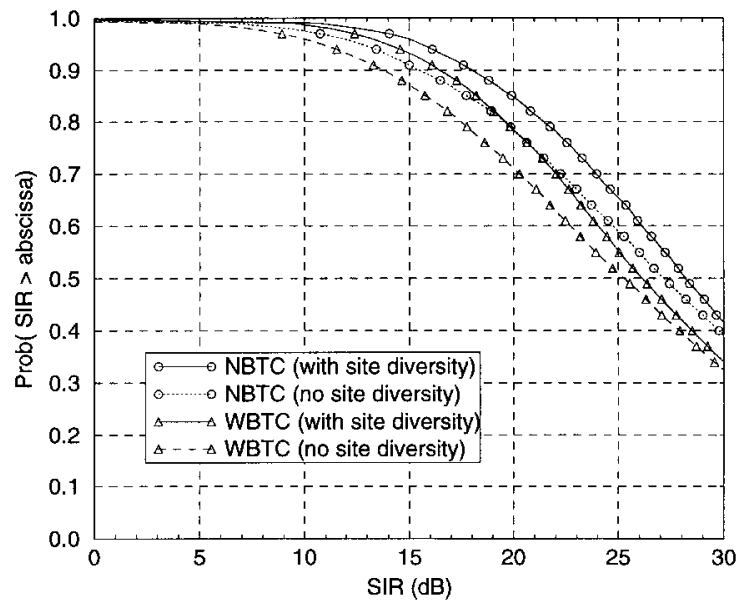

Fig. 14. Site diversity gain for the NBTC and WBTC systems in terms of SIR performance improvement, where $N=4$ and $\sigma=6 \mathrm{~dB}$.

the WBTC system both with and without site diversity. For the NBTC system with diversity, the 90th-percentile value of SIR is $1.5 \mathrm{~dB}$ better than that of the WBTC system; without diversity, the performance difference is $2.5 \mathrm{~dB}$. The SIR improvement may lead to an increase in system capacity. For example, if the SIR requirement is a 90 th percentile equal to $17 \mathrm{~dB}$, then only the NBTC system is capable of using $N=4$.

Table II lists the 90th-percentile value of SIR for the NBTC and WBTC systems for other combinations of $N$ and $\sigma$. Comparing the SIR performance of the two systems without site diversity (Table I) and with site diversity (Table II), we observe the following: (1) for the values of $\sigma$ and $N$ considered, the site diversity gains range from around 0.9 to $6.2 \mathrm{~dB}$ for both systems; (2) the larger the value of shadow-fading standard deviation, the larger the site diversity gain; (3) the site diversity gains are greater for the WBTC system; (4) as a consequence, the improvements of NBTC systems over WBTC systems shrink, with site diversity, to values ranging from

Table II. The 90th-Percentile SIR for NBTC and WBTC Systems with Site Diversity

\begin{tabular}{cccccccc}
\hline & \multicolumn{3}{c}{ NBTC } & & \multicolumn{3}{c}{ WBTC } \\
\cline { 2 - 4 }$\sigma(\mathrm{dB})$ & $N=3$ & $N=4$ & $N=7$ & $N=3$ & $N=4$ & $N=7$ \\
\hline 4 & 17.99 & 20.26 & 24.77 & 16.49 & 18.84 & 23.59 \\
6 & 15.91 & 18.02 & 22.70 & & 14.21 & 16.46 & 21.38 \\
8 & 13.94 & 16.08 & 20.76 & & 11.68 & 13.81 & 18.78 \\
10 & 11.97 & 14.02 & 18.70 & 8.77 & 10.93 & 16.01 \\
\hline
\end{tabular}


1.2 to $3.2 \mathrm{~dB}$; and (5) the improvement of NBTC systems over WBTC systems increases with shadow-fading standard deviation and decreases with reuse factor.

Figure 15 shows the coverage performance for both systems for $\sigma=6 \mathrm{~dB}$. Observe that with or without site diversity, at the $p=10 \%$ level, the NBTC systems outperforms the WBTC system by around $2 \mathrm{~dB}$. This improvement implies a decrease in the required transmit power; alternatively, assuming the same transmit power, it implies a larger cell coverage area, and therefore a smaller number of cells for the NBTC system.

Table III summarizes the coverage performances at the $p=10 \%$ level of the two systems with and without site diversity. We found that, for $\sigma=4 \sim 10 \mathrm{~dB}$, the coverage performance gain as a result of site diversity is almost the same for the two systems. Therefore, the table just includes one column for site diversity gain. The table also lists the difference in transmit power requirements between the two systems. We observe the following: (1) for $\sigma=4 \sim 10 \mathrm{~dB}$, the site diversity gains range from 1.5 to $7.0 \mathrm{~dB}$ for both systems, almost the same range as that for the corresponding SIR improvement; (2) as may have been predicted, the larger the value of shadow standard deviation, the larger the site diversity gain; and (3) just as for SIR, an NBTC system consistently outperform s a WBTC system, with the gain increasing from 2 to $3 \mathrm{~dB}$ as $\sigma$ increases from 4 to $10 \mathrm{~dB}$. For reference, if $\gamma=4$, then a $2.5 \mathrm{~dB}$ gain in transmit power translates to a $33 \%$ increase in cell area, or equivalently, to a $25 \%$ reduction in the number of base stations required to cover a given area.

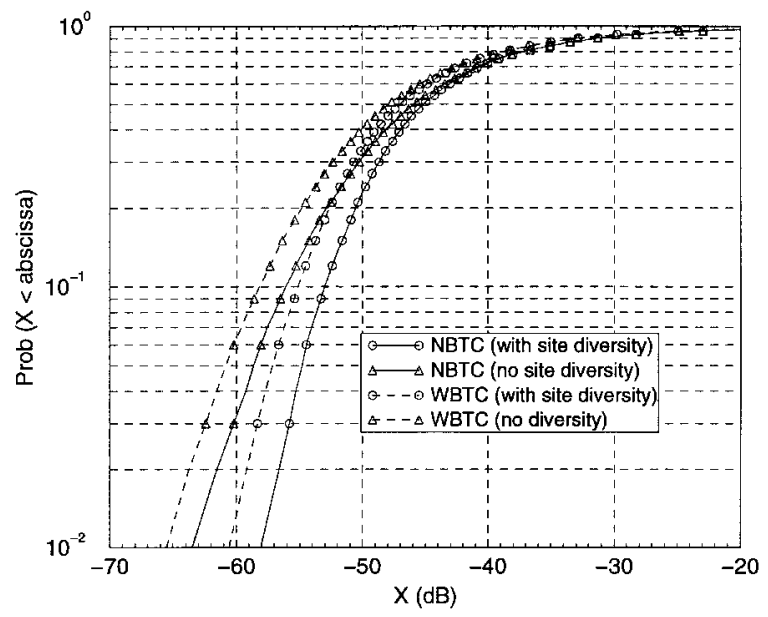

Fig. 15. Coverage performance of NBTC and WBTC systems with $\gamma$ $=4$ and $\sigma=6 \mathrm{~dB}$.
Table III. Coverage Performance Improvements: Site Diversity and NBTC System

\begin{tabular}{ccc}
$\sigma(\mathrm{dB})$ & $\begin{array}{c}\text { Site Diversity } \\
\text { Gain }(\mathrm{dB})\end{array}$ & $\begin{array}{c}\text { Transmit Power Improvement } \\
\text { of NBTC }(\mathrm{dB})\end{array}$ \\
\hline 4 & 1.6 & 2.0 \\
6 & 3.1 & 2.2 \\
8 & 4.8 & 2.3 \\
10 & 7.0 & 3.0 \\
\hline
\end{tabular}

\section{SENSITIVITY TO LOCATION VARIATION}

So far we have assumed that the cell sites are in their ideal locations, i.e., cell site locations are available everywhere. However, this is seldom true in practice. This section discusses the sensitivity of the NBTC and WBTC systems to site location variations. We do not consider the sensitivity to errors in antenna pointing since it is easier to align the direction perfectly than to choose the site location.

We model the location variation by assuming that each base lies within a circle of radius $\eta R$ centered on its ideal location, where $R$ is the cell radius and $\eta$ is a parameter. The position of each base within its permissible circle of radius $\eta R$ is uniformly distributed in this circle. We obtain simulation results as $\eta$ varies from 0 (ideal-grid case) to 0.5 .

\subsection{Simulation Approach}

1. For a given value of $\eta$, randomly generate the cell site locations within their permissible circles.

2. Evaluate the SIR and coverage performance (NBTC and WBTC) with site diversity for $N=$ $4, \sigma=6 \mathrm{~dB}$, and $\gamma=4$, for all mobiles served by a specific sector.

3. Repeat Steps 1 and 2 for 50 different trials.

4. Do the above for $\eta=0.1,0.2,0.3,0.4$, and 0.5 .

\subsection{Numerical Results}

Figures 16 and 17 show the SIR performances of the NBTC and WBTC systems for $\eta=0.2$; Figs. 18 and 19 show the corresponding coverage performance. In each figure, the nominal case corresponds to ideal cell site location, and the worst case refers to the worst observed performance over the 50 trials. We see from Figs. 16 and 17 that for $\eta=0.2$, the maximum degradation of the 90th-percentile value of the SIR, for either 


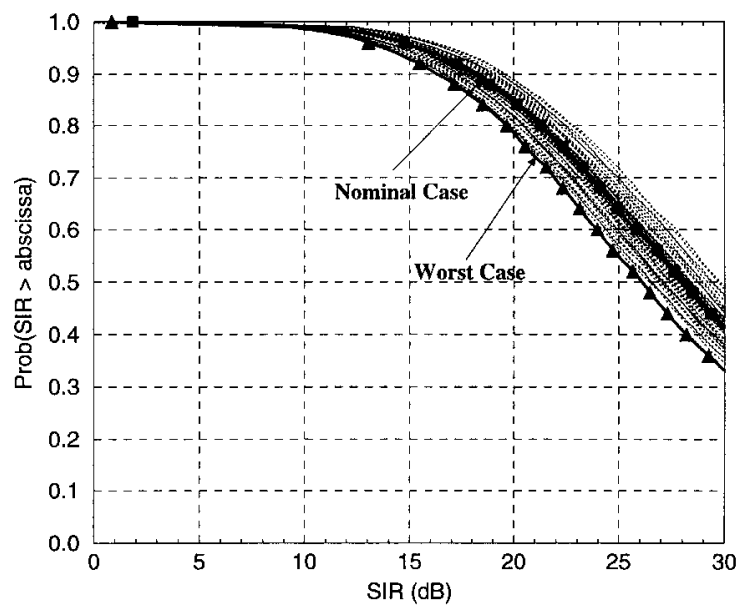

Fig. 16. Sensitivity of SIR performance on variation of cell site location for NBTC systems with site diversity, where $\eta=0.2$, the ratio of the radius of site uncertainty area to a cell radius; $N=4 ; \sigma=6 \mathrm{~dB}$.

system, is less than $2 \mathrm{~dB}$. From Figs. 18 and 19, we see that the maximum degradation in the coverage performance (10th percentile of $X$ ), for either system, is around $1.5 \mathrm{~dB}$.

Table IV summarizes the sensitivity of the SIR performance to location variation in terms of the mean and the standard deviation, over the 50 trials, of the 90thpercentile SIR. From the table, we see that (1) for the values of $\eta$ considered, the mean performance of the NBTC system continues to be better than the mean performance of the WBTC system, even though the perfor-

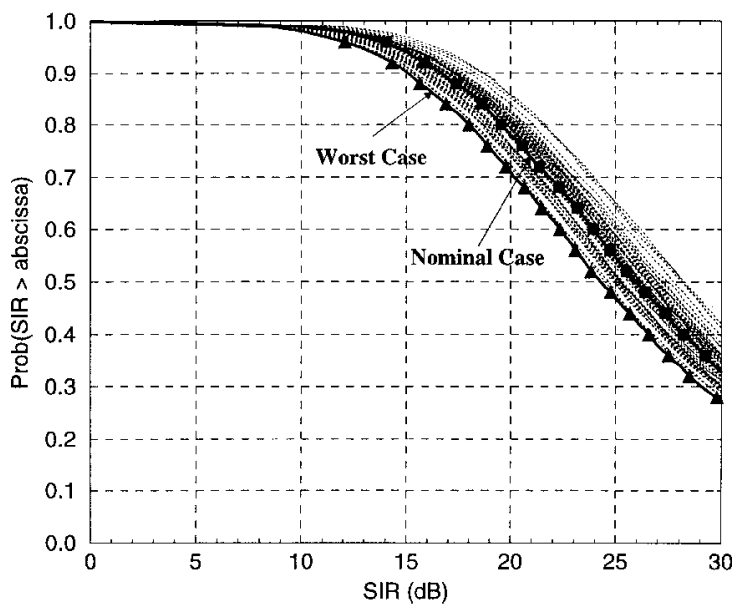

Fig. 17. Sensitivity of SIR performance on variation of cell site location for WBTC systems with site diversity, where $\eta=0.2$, the ratio of the radius of site uncertainty area to a cell radius; $N=4 ; \sigma=6 \mathrm{~dB}$.

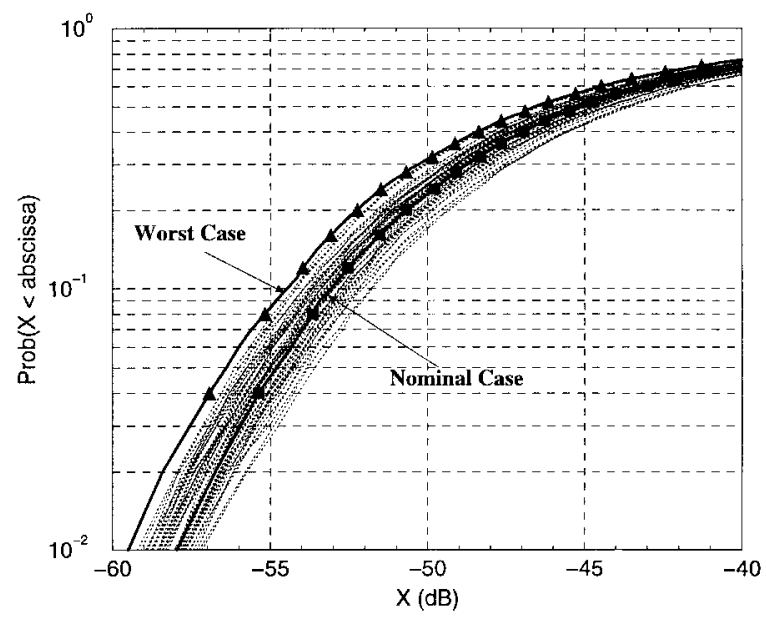

Fig. 18. Sensitivity of RSS performance on variation of cell site location for NBTC systems with site diversity, where $\eta=20 \%$, the ratio of radius of uncertainty area for a cell site to that of a cell coverage area; $N=4, \sigma=6 \mathrm{~dB}$.

mance gap narrows from 1.4 to $0.8 \mathrm{~dB}$ as $\eta$ increases from 0.1 to 0.5 ; (2) the standard deviations (computed in the $\mathrm{dB}$ domain) of the SIR of the two systems are close for all values of $\eta$, and increase as $\eta$ increases. This phenomenon also tends to narrow the performance gap between the two systems with increasing $\eta$.

Table V summarizes the sensitivity of the coverage performance to location variation, in terms of the mean and the standard deviation, over the 50 trials, of

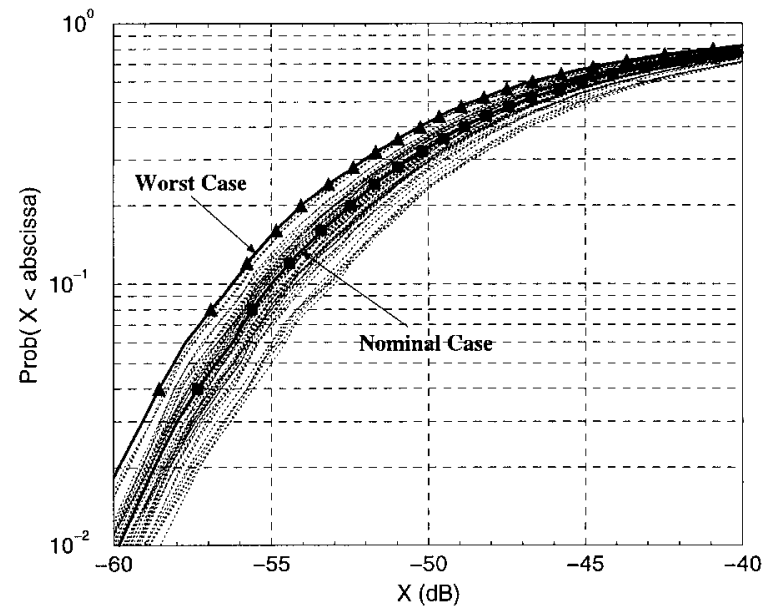

Fig. 19. Sensitivity of RSS performance on variation of cell site location for WBTC systems with site diversity, where $\eta=20 \%$, the ratio of radius of uncertainty area for a cell site to that of a cell coverage area; $N=4 ; \sigma=6 \mathrm{~dB}$. 
Table IV. The Mean and Standard Deviation of the 90th-Percentile Values of SIR

\begin{tabular}{|c|c|c|c|c|}
\hline \multirow[b]{2}{*}{$\eta$} & \multicolumn{2}{|c|}{ NBTC } & \multicolumn{2}{|c|}{ WBTC } \\
\hline & Mean & $\begin{array}{c}\text { Standard } \\
\text { Deviation }\end{array}$ & Mean & $\begin{array}{l}\text { Standard } \\
\text { Deviation }\end{array}$ \\
\hline 0.1 & 18.09 & 0.51 & 16.71 & 0.41 \\
\hline 0.2 & 18.05 & 0.90 & 16.79 & 0.87 \\
\hline 0.3 & 17.94 & 1.24 & 16.84 & 1.23 \\
\hline 0.4 & 17.74 & 1.48 & 16.82 & 1.57 \\
\hline 0.5 & 17.41 & 1.70 & 16.64 & 1.79 \\
\hline
\end{tabular}

the 90th-percentile $X$. From the table, we see that (1) as for SIR, the mean coverage performance of the NBTC system continues to be better than the mean coverage performance of the WBTC system, and this performance gap narrows from 1.9 to $0.8 \mathrm{~dB}$ as $\eta$ increases from 0.1 to 0.5 ; (2) the standard deviations (computed in the $\mathrm{dB}$ domain) of the coverage performance $(X)$ of the two systems increase as $\eta$ increases, again leading to a narrowing of the performance gap; (3) the standard deviations of the coverage performance $(X)$ of the NBTC systems are less than those of the WBTC systems.

Table VI lists the worst-case 90th-percentile SIR over the 50 trials, for different values of $\eta$. From the table, we see that the worst-case performance of the NBTC system continues to be better than that of the WBTC system, even though the performance gap narrows from 1.4 to $0.6 \mathrm{~dB}$ as $\eta$ increases from 0.1 to 0.5 ; The table also lists the worst-case performance degradation ("Loss") of either system as $\eta$ varies from 0.1 to 0.5 . We see from the table that for $\eta=0.5$, the 90thpercentile SIR can be as much as $4.2 \mathrm{~dB}$ below the

Table V. The Mean and Standard Deviation of the 10th-Percentile Values of $X$

\begin{tabular}{lccccc}
\hline & \multicolumn{2}{c}{ NBTC } & & \multicolumn{2}{c}{ WBTC } \\
\cline { 2 - 3 } \cline { 5 - 6 }$\eta$ & Mean & $\begin{array}{c}\text { Standard } \\
\text { Deviation }\end{array}$ & & Mean & $\begin{array}{c}\text { Standard } \\
\text { Deviation }\end{array}$ \\
\hline 0.1 & -53.03 & 0.37 & -54.92 & 0.38 \\
0.2 & -53.14 & 0.70 & & -54.76 & 0.80 \\
0.3 & -53.31 & 0.94 & & -54.66 & 1.19 \\
0.4 & -53.53 & 1.11 & & -54.62 & 1.54 \\
0.5 & -53.93 & 1.27 & -54.75 & 1.77 \\
\hline
\end{tabular}

Table VI. The Performance Degradations of NBTC Systems and WBTC Systems Due to the Location Variations of Cell Sites, in Terms of Worst-Case 90th-Percentile SIR

\begin{tabular}{lcccccc}
\hline & \multicolumn{2}{c}{ NBTC } & & \multicolumn{2}{c}{ WBTC } & \\
\cline { 2 - 3 }$\eta$ & (A) & Loss & & (B) & Loss & $(\mathrm{A})-(\mathrm{B})$ \\
\hline 0 & 18.02 & - & & 16.46 & - & 1.56 \\
0.1 & 17.07 & 0.95 & & 15.72 & 0.74 & 1.35 \\
0.2 & 16.30 & 1.72 & 15.09 & 1.37 & 1.21 \\
0.3 & 15.15 & 2.87 & 14.17 & 2.29 & 0.98 \\
0.4 & 14.51 & 3.51 & 13.77 & 2.69 & 0.74 \\
0.5 & 13.83 & 4.19 & 13.21 & 3.21 & 0.62 \\
\hline
\end{tabular}

nominal value in an NBTC system, and as much as 3.2 $\mathrm{dB}$ below the nominal value in a WBTC system. Usually $\eta=0.1-0.25$ is the typical range $[9,12]$.

Table VII shows the corresponding worst-case coverage performance, i.e., it lists the 10th-percentile $X$ over the 50 trials, for different values of $\eta$. From the table, we see that, as for SIR, the worst-case performance of the NBTC system continues to be better than that of the WBTC system, even though the performance gap narrows from 1.8 to $1.0 \mathrm{~dB}$ as $\eta$ increases from 0.1 to 0.5 . The table also lists the worst-case performance degradation ("Loss") of either system as $\eta$ varies from 0.1 to 0.5 . We see from the table that for $\eta=0.5$, the 10 th-percentile $X$ can be as much as $3.9 \mathrm{~dB}$ below the nominal value in an NBTC system, and as much as $3.1 \mathrm{~dB}$ below the nominal value in a WBTC system.

To summarize, we find that NBTC systems continue to outperform WBTC systems, in terms of both SIR and coverage, even for nonideal locations of cell sites. However, the performance gaps narrow as the cell sites are

Table VII. The Performance Degradations of NBTC Systems and WBTC Systems Due to the Location Variations of Cell Sites, in Terms of Worst-Case 10th-Percentile $X$

\begin{tabular}{lcccccc}
\hline & \multicolumn{2}{c}{ NBTC } & & \multicolumn{2}{c}{ WBTC } & \\
\cline { 2 - 3 }$\eta$ & (A) & Loss & & (B) & Loss & (A) $-(B)$ \\
\hline 0 & -53.08 & - & & -55.01 & - & 1.93 \\
0.1 & -53.77 & 0.69 & & -55.59 & 0.58 & 1.82 \\
0.2 & -54.49 & 1.41 & & -56.26 & 1.25 & 1.77 \\
0.3 & -55.35 & 2.27 & & -56.96 & 1.95 & 1.61 \\
0.4 & -56.16 & 3.08 & & -57.58 & 2.57 & 1.42 \\
0.5 & -57.02 & 3.94 & & -58.08 & 3.07 & 1.06 \\
\hline
\end{tabular}


allowed to be located farther away from their ideal locations.

\section{CONCLUDING REMARKS}

We have used Monte Carlo simulations to assess two trisector cellular architectures: the traditional one using $100^{\circ}-120^{\circ}$ antenna beams (and called WBTC herein) and the newer clover-leaf architecture using $60^{\circ}-70^{\circ}$ beams (and called NBTC herein). For each, we have quantified the statistics of both downlink signal-tointerference ratio and received downlink signal power. In so doing, we have considered the effects of (1) channel utilization factor, (2) site diversity, (3) nonideal cell site locations, and (4) the influences of reuse factor $(N)$ and shadow fading standard deviation $(\sigma)$. We have also examined to a limited extent the effects of antenna pattern and the path loss exponent $(\gamma)$. Although we have not analyzed the uplink, we would expect the comparisons for the uplink to be close to those for the downlink.

We would like to point out that because of handoff hysteresis or call dragging, and also because of measurement errors, the performance results assuming site diversity are somewhat optimistic. Therefore, it is useful to also present the performance results without site diversity, as we have done. On the other hand, because we did not include any fine-tuning (altering the power settings, for example) in the study on site location variations, the results for this case are somewhat pessimistic. Notwithstanding these comments, we expect that the performance results obtained are representative of the two architectures, and especially so as concerns the comparison between them.

We conclude, therefore, that NBTC system performance exceeds that of WBTC systems, both in SIR statistics and signal coverage. The improvement in coverage translates to a reduced power requirement of about $2 \mathrm{~dB}$ for the same cell size, or equivalently to a $25 \%$ larger cell coverage area (assuming $\gamma=4$ ) for the same power. The improvement in SIR performance corresponds to a gain (for typical values of $\sigma$ ) of 2 to $3 \mathrm{~dB}$ in the 90th SIR percentile. Whether this gain can be exploited to increase reuse efficiency (and thus capacity) is not clear. It certainly can be translated to significantly improve grade of service in cellular environments, with no attendant cost.
As regards future work, there are at least two directions worth considering. The first is to model terminal mobility and system latency, and then reevaluate the performance of each architecture. The second is to study the performance for spatially nonuniform traffic. We do not expect these studies to change our main conclusion, but we do expect to be able to provide even more realistic performance evaluations.

\section{ACKNOWLEDGMENTS}

The authors would like to thank Jim Whitehead for providing valuable comments and Martin Clark for his help with the radiation patterns of directional antennas.

\section{NOTES}

1. We assume here that the user's antenna is omnidirectional with respect to azimuth angle $\theta$.

2. For studying SIR performance, we ignore the effects of receiver thermal noise.

3. Hereafter, we deal only with $\mathrm{dB}$ values of signal-to-interf erence ratio.

\section{REFERENCES}

1. V. Palestini, Alternative frequency plans in hexagonal-shaped cellular layouts, in IEEE International Symposium on Personal, Indoors, and Mobile Radio Communications, 1992, pp. 585-590.

2. W. C. Y. Lee, Smaller cells for greater performance, IEEE Communications Magazine, Nov. 1991, pp. 19-23.

3. Y. S. Yeh and S. C. Schwartz, Outage probability in mobile telephone due to multiple log-normal interferers, IEEE Transactions on Communications, Vol. 32, Apr. 1984, pp. 380-388.

4. C. Balanis, Antenna Theory. Harper \& Row, New York, 1982.

5. G. C. D. Piazza, A. Plitkins, and G. I. Zysman, The cellular test bed, The Bell System Technical Journal, Vol. 58, Jan. 1979, pp. 215-248.

6. Product Selection Guide 195, CELWAVE Inc., 1995.

7. Wireless Communication Antennas Catalog. Radiation Systems Inc., 1995.

8. M. V. Clark, V. Erceg, and L. J. Greenstein, Reuse efficiency in urban microcellular networks, in IEEE Vehicular Technology Conference, 1996, pp. 421-425.

9. R. Rudokas and T. Benz, Is it time to rethink frequency reuse formulas? Telephony, Vol. 232, Mar. 1997, pp. 68-74.

10. S. Ariyavisitakul, T. E. Darcie, L. J. Greenstein, M. P. Phillips, and N. K. Shankaranarayanan, Performance of simulcast wireless techniques for personal communication systems, IEEE Journal on Selected Areas in Communication, Vol. 14, May 1996, pp. 632-643.

11. L. C. Wang and C. T. Lea, Macrodiversity cochannel interference analysis, Electronics Letters, Vol. 31, Apr. 1995, pp. 614-616.

12. Personal communications with J. F. Whitehead, Feb. 1997. 


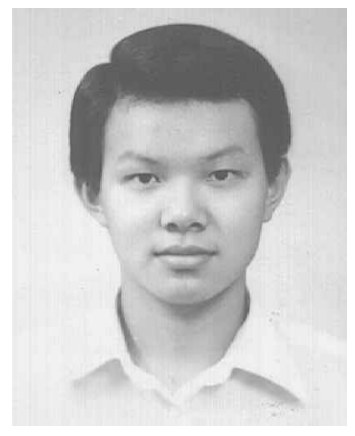

Li-Chun Wang received the B.S. degree from National Chiao Tung University in 1986; the M.S. degree from National Taiwan University in 1988; the M.Sci. and Ph.D. degrees from the Georgia Institute of Technology in 1995 and 1996, all in electrical engineering. From 1990 to 1992 he was with the Telecommunication s Laboratories of Ministry of Transportations and Communications in Taiwan (currently the Telecom Labs of Chunghwa Telecom Co.). In 1995 he worked at Bell Northern Research of Northern Telecom Inc. (currently Nortel Inc.) in Richardson, Texas. Since July 1996, Dr. Wang has been with AT\&T Laboratories, where he is a Senior Technical Staff Member in the Wireless Communications Research Department. His current research interests are in the area of cellular architectures, radio resource management, and propagation channel modeling. Specific topics include hierarchical cellular architectures, macrodiversity cellular systems, dynamic channel allocations, power control, and microcellular interference modeling.

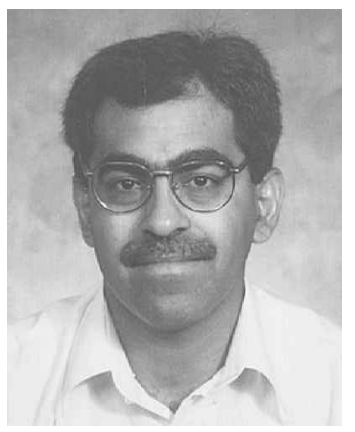

Kapil Chawla received the $\mathrm{B}$. Tech. degree from the Indian Institute of Technology, Kanpur, in 1984, and the M.S. and Ph.D. degrees from the University of Illinois at Urbana-Champaign in 1987 and 1991, all in electrical engineering. He joined AT\&T Bell Laboratories in November 1990, where he worked on several aspects of wireless and wireline communications, including systems engineering, performance analysis, and network planning. He is currently with the Wireless Communications Research Department of AT\&T Laboratories, Red Bank, New Jersey. His research interests are in the general areas of resource assignment in wireless networks and cellular system engineering.

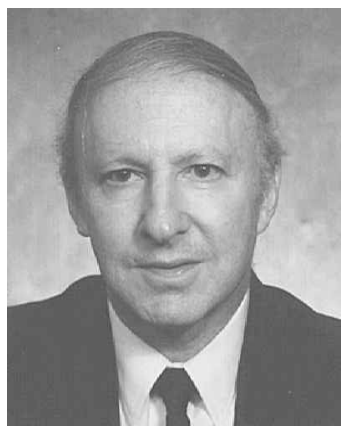

Larry J. Greenstein received the B.S., M.S., and Ph.D. degrees in electrical engineering from Illinois Institute of Technology in 1958, 1961, and 1967, respectively. From 1958 to 1970, he worked at IIT Research Institute, primarily in the areas of radio frequency interference and anticlutter airborne radar. He joined AT\&T (then Bell Telephone Laboratories) in 1970 and has conducted research there in communications satellites, microwave digital radio, lightwave transmission, and wireless communications. He currently heads the Wireless Communications Research Department of AT\&T Labs-Research. His areas of concentration in the wireless field include measurementbased propagation modeling of both fixed and mobile channels, microcell system engineering, techniques for diversity and equalization, and methods for assessing and optimizing system capacity and performance. Dr. Greenstein was co-recipient of the IEEE Communications Society's 1984 Leonard G. Abraham Prize Paper Award and the IEEE Vehicular Technology Society's 1993 Neal Shepard Prize Paper Award. $\mathrm{He}$ also co-authored the IEEE Press reprint book Microwave Digital Radio. He has been a guest editor, senior editor, and editorial board member for numerous publications, and has organized and chaired technical sessions for a number of international conferences. 